\title{
Relato de uma Experiência de Ensino de Vigilância à Saúde para Residentes de Pediatria
}

\author{
Selma Cristina Franco \\ Emília de Faria Carniel \\ Maria Ângela R. G. M. Antônio' \\ Maria de Lurdes Zanolli"
}

\begin{abstract}
RESUMO
Um dos enfoques mais importantes na reoriertação da formação profissional do médico nas últimas décadas tem sido a articulação do ensino com os serviços de saúde, trazendo contribuiç̧̃es para a construção de um novo modelo assistencial e, ao mesmo tempo, oferecendo à universidade um campo de pesquisa e ensino. Os autores relatam uma experiência de ensino de Vigilância à Saúde, com a inserção de residentes de Pediatria em uma Rede Municipal de Saúde, onde realizam assistência à saúde de crianças e adolescentes, sob supervisão docente. O estágio passou por diversas reformulações desde sua implantação, acompanhando todo o processo de reforma do setor saúde, sempre buscando propostas mais adequadas de ensino-aprendizagem. Os autores expōem os objetivos da proposta de estágio, descrevem as estratégias didáticas utilizadas, apresentam os resultados das atividades realizadas e analisam o impacto sobre o ensino e o serviço de saúde, com críticas e propostas de reformulações.
\end{abstract}

\section{INTRODUÇÃO}

Os debates sobre educação médica presentes na atualidade em instituições de ensino, entidades profissionais, órgãos governamentais e sociedade em geral refletem a preocupação existente em muitos países com a qualidade dos profissionais médicos formados nas últimas décadas. Desde o Relatório Flexner (1910), inúmeras constatações de deficiências na formação médica vêm sendo apontadas, entre elas o modelo biologicista, que privilegia as especialidades e é fortemente centrado em procedimentos de alta tecnologia ${ }^{1,2}$.

O perfil de profissional formado, refletindo as distorções de tal modelo pedagógico, caracterizou-se por uma prática clínica centrada em aspectos biológicos do indivíduo doente, desvinculado de

\section{DESCRITORFS}

- Vigilância sanitária;

Estágio clínico-tendências;

Corpo clínico hospitalar;

- Assistentes de Pediatriaeducação;

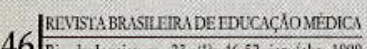

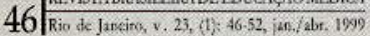
seu contexto sócio-cultural, priorizando a técnica em detrimento de um enfoque humanístico na relação médico-paciente e com pouca ênfase na prevenção e promoção da saúde.

O ensino e treinamento destes profissionais centrados nos Hospitais Universitários, e distantes dos demais níveis de atenção, impossibilitou-os de observar e vivenciar os problemas de saúde mais prevalentes em termos epidemiológicos e a questão da inserção social determinando a qualidade de vida e saúde das pessoas ${ }^{3}$.

Este distanciamento entre ensino e realidade levou a prática médica a adquirir contornos dife-

1 Médica pediatra e sanitarista do Departamento de Pediatria da FCM/Unicamp.

2 Enfermeira do Departamento de Pediatria da FCM/Unicamp.

3 Professora Assistente do Departamento de Pediatrin FCM/Unicamp. 
rentes em cada país, em função de suas diversas características sociais. Nos países desenvolvidos, enfatizou-se a tecnicalidade da prática e as dificuldades na relação médico-paciente. Assim, as propostas para superar estes problemas voltaram-se para treinamentos e reformas curriculares para modificação de atitudes e aquisição de habilidades visando melhorar a interação médico-paciente ${ }^{4,5}$, atenção orientada para a comunidade ${ }^{6,7}$, mudanças de enfoque nas avaliações ${ }^{8,9}$, ensino baseado em evidências e enfoque interdisciplinar ${ }^{10}$. Todas elas têm em comum o fato de promoverem mudanças de comportamento pontuais e com caráter mais periférico, verdadeiros apêndices no conjunto curricular da maioria das escolas médicas ${ }^{11}$.

No Brasil, bem como em outros países subdesenvolvidos, somou-se a estas características o desconhecimento de suas realidades sanitárias, prejudicando a compreensão e atuação efetiva sobre os determinantes do processo saúde-doença ${ }^{12}$.

A constatação da inadequação do profissional médico para atender às demandas sociais de nossa população levou as escolas médicas a proporem uma série de reformulações curriculares buscando inserir o ensino no contexto da realidade e dos serviços de saúde.

Em 1972, reconhecendo a estreita ligação entre a organização dos cuidados à saúde e o ensino médico, a OMS afirmou que "a educação médica deve estar intrinsecamente ligada ao sistema de saúde e, dessa forma, quando surgirem questões sobre o sistema de saúde, questões sobre educação médica devem se seguir". Entretanto, em nosso país, a reestruturação do sistema de atenção à saúde foi alicerçada sobre o movimento da Reforma Sanitária, que ocorreu no interior do movimento geral pela redemocratização do país e fora do âmbito das instituições de ensino ${ }^{13,14}$.

A adesão das escolas médicas ao movimento foi parcial, limitada aos Departamentos de Medicina Preventiva e excepcionalmente aos Departamentos de Pediatria, Tocoginecologia e Psiquiatria, que reconheceram na instalação da rede básica de saúde um campo potencial de aprendizagem para seus alunos. Os projetos de Integração Docente-Assistencial (IDA), surgidos no final da década de 70 e início da década de 80 , são exemplos de iniciativas neste sentido ${ }^{14,15,16}$. Porém, o caráter de extensão destes projetos, complementando o modelo hegemônico, não foi suficiente para produzir o impacto esperado sobre o perfil do profissional formado.
No início dos anos 90, surgiram os projetos UNI, em diversos países da América Latina e Caribe, apoiados por iniciativas internacionais, articulando universidade, serviços e comunidades no processo de construção dos sistemas locais de saúde ${ }^{14,15}$.

Assim, em todos estes projetos, a inserção das escolas médicas nos serviços de saúde, participando da construção do sistema de saúde, deu-se de modo parcial e, conseqüentemente, a formação profissional modificou-se muito pouco nestes anos. Enquanto as propostas em relação à reestruturação do sistema de saúde avançaram nestas duas décadas, a universidade resistiu a reformular-se, limitando-se a propor reformas curriculares que, de fato, não produziram mudanças significativas na formação do profissional médico ${ }^{16}$. A instituição acadêmica e seus membros foram apontados como os principais responsáveis pela resistência às mudanças ${ }^{5,6,11}$.

Diante deste quadro, nos últimos anos, surgiram numerosas propostas de reformas e avaliações curriculares, merecendo destaque o projeto da Cinaem (Comissão Interinstitucional Nacional de Avaliação do Ensino Médico), iniciado em 1991, com adesão de 48 escolas e cujos resultados possibilitaram a formulação de diversas propostas para transformar o ensino médico ${ }^{17,18}$.

O relato de experiências de inserção de alunos de Medicina nos serviços básicos de saúde, como parte de modificações da grade curricular, limita-se a poucos periódicos das próprias escolas médicas, de algumas entidades internacionais (Opas, OMS) ou daquelas diretamente envolvidas com estes projetos, não circulando de maneira mais global. Algumas destas experiências, desenvolvidas no campo da Pediatria, foram altamente positivas do ponto de vista tanto de alunos e docentes quanto dos serviços nos quais eles se inseriram ${ }^{19,20,21}$.

\section{A ELABORAÇÃO DE UMA PROPOSTA}

Entendendo que a articulação do ensino com os serviços de saúde contribui para a construção de um novo modelo assistencial e, ao mesmo tempo, oferece à universidade mais um campo de pesquisa e ensino, o Departamento de Pediatria da FCM-Unicamp, por meio da equipe de profissionais do Setor de Pediatria Social (atualmente composta por pediatras e uma enfermeira), atua há cerca de 20 anos com alunos de Me- 
dicina e residentes de Pediatria, inserindo-os na Rede Básica de Saúde do município de Campinas (SP), onde desenvolvem atividades de assistência à criança, sob supervisão docente, dando continuidade às atuações que este Departamento desenvolveu na década de 60 na Rede Municipal e no Centro de Saúde Escola de Paulínia, e que foram integradas com o Departamento de Medicina Preventiva e Social a partir da década de 70. A viabilização deste projeto foi possível por uma série de motivos, entre os quais a adesão de gestores municipais ao movimento de reestruturação dos serviços de saúde, possibilitando a criação da rede municipal de saúde na década de 70 .

Diversas reformulações no estágio vêm sendo feitas nestes anos, acompanhando todo o processo de reforma do setor saúde e sempre buscando propostas mais adequadas de ensino-aprendizagem. Há dois anos foi possível operacionalizar, dentro do estágio da Pediatria Social, o ensino de Vigilância à Saúde da criança com ações na comunidade, para alunos de quarto ano médico e residentes de primeiro ano de Pediatria em uma Unidade Básica de Saúde (UBS) do município de Campinas.

\section{OBJETIVO}

O objetivo geral deste artigo é relatar a experiência de ensino em Vigilância à Saúde para residentes de primeiro ano de Pediatria.

\section{DESCRIÇÃO DA PROPOSTA DE ENSINO DE VIGILÂNCIA À SAÚDE}

\section{CONCEITUAÇÃO}

Considerando-se a existência de diferentes vertentes na concepção da Vigilância, expressas nas diversas terminologias utilizadas, como "Vigilância da Saúde", "Vigilância à Saúde", "Vigilância em Saúde" optaram pela concepção materializada por meio da Lei $n^{\circ}$ 791/95, que estabelece o Código de Saúde no Estado de São Paulo. Nela reconhece-se a interdependência de conteúdo e de desenvolvimento nas ações das vigilâncias sanitária e epidemiológica, integrando-as conceitualmente sob o nome de Vigilância à Saúde, que pode ser traduzida como um "compromisso em defesa da qualidade de vida" ${ }^{23}$.

\section{OBJETIVOS DIDÁTICOS}

O objetivo geral do estágio é possibilitar aos residentes a compreensão da inserção social dos indivíduos como determinante de sua qualidade de vida e saúde.

Os objetivos específicos são:

- Compreender a Vigilância à Saúde como uma prática realizada pelos serviços de saúde objetivando minimizar os efeitos de determinantes do processo saúde-doença;

- Vivenciar as ações em saúde coletiva e atuar em situações ligadas à Vigilância Epidemiológica e Sanitária, buscando integrá-las dentro do conceito mais abrangente de Vigilância à Saúde;

- Conhecer a relação das doenças sujeitas a notificação compulsória no Município de Campinas (SP) e seus respectivos manuais e normas técnicas;

- Produzir e analisar boletins epidemiológicos a partir de dados do serviço;

- Entender a organização do serviço de Vigilância à Saúde em uma Unidade Básica de Saúde (UBS) e suas formas de atuação;

- Compreender as atribuições dos serviços de vigilância regionais e hospitalares e sua articulação com os demais serviços de diferentes níveis hierárquicos;

- Conhecer o fluxo de informações nos diversos níveis do sistema de saúde e sua integração.

\section{ESTRATÉGIAS DIDÁTICAS}

Para atingir os objetivos expostos, utilizou-se a seguinte estratégia didática: durante o estágio no Setor de Pediatria Social, grupos de dois a três residentes fizeram rodízio a cada cinco semanas, desenvolvendo uma vez na semana as atividades propostas de Vigilância à Saúde, sob a supervisão de um ou dois docentes responsáveis, que são três pediatras e uma enfermeira, num total de 50 horas. A área geográfica de atuação corresponde à cobertura de uma Unidade Básica de Saúde (UBS) onde a equipe da Pediatria Social desenvolve seu estágio com alunos do curso médico desde 1995. 
Foram realizadas as seguintes atividades:

- Discussões teóricas com os seguintes conteúdos: aspectos históricos da Vigilância no Brasil, suas reformulações acompanhando as modificações do Sistema Único de Saúde e suas perspectivas atuais; sistemática de uma investigação epidemiológica; intervenções propostas para a Vigilância à Saúde, exemplificadas por meio de discussão dos manuais das doenças sujeitas a notificação compulsória de maior prevalência em nosso meio (12 horas);

- Preparo e realização de visitas em domicílios e instituições, dirigidas para os casos de doença de notificação compulsória ocorridos dentro da área de cobertura da UBS, além de casos selecionados a partir de critérios de risco (biológico, psicológico ou social). Vale ressaltar que o preparo desta atividade permitia flexibilidade para adaptações em decorrência de situações momentâneas que fossem de interesse para o aprendizado, como surtos de doenças infecto-contagiosas, operação arrastão para combate ao dengue, etc. (25 horas);

- Confecção de relatórios imediatamente após cada uma destas atividades, anotando-se em prontuários do serviço os aspectos relevantes de cada caso, enfatizando os limites e possibilidades de atuação dos serviços de saúde sobre os determinantes do processo saúde-doença, no sentido de preservar a saúde das crianças (5 horas);

- Visitas a um Núcleo de Vigilância Epidemiológica Hospitalar e a um Núcleo Regional de Vigilância do município, para conhecer suas respectivas atribuições dentro da rede hierarquizada de atenção à saúde e sua integração com os demais niveis (4 horas);

- Notificação das doenças ocorridas na área de cobertura da UBS na semana epidemiológica anterior e envio dos respectivos impressos, obedecendo à normatização proposta para o fluxo de papéis (1 hora);

- Organização do banco de dados do serviço por meio da confecção de boletins epidemiológicos ao final de cada rodízio (3 horas).

As atividades desenvolvidas entre janeiro/96 e janeiro/98 foram dispostas na Tabela 1, que exemplifica os diversos tipos de intervenções que o serviço de saúde realizou de acordo com as peculiaridades de cada agravo e demonstra o potencial que a UBS oferece como campo de ensino-aprendizagem de Vigilância à Saúde.

TABELA 1

Atividades de Vigilância à Saúde em relação às situações/agravos realizadas de janeiro/96 a janeiro/98

\begin{tabular}{|c|c|c|c|c|c|c|c|c|}
\hline DOENÇAS & $\begin{array}{c}\text { VISITAS } \\
\text { PROGRAMADAS } \\
\text { (N) } \\
\end{array}$ & $\begin{array}{l}\text { VISITAS } \\
\text { REALIZADAS } \\
\text { (N) }\end{array}$ & $\begin{array}{c}\text { PACIENTES } \\
\text { CONVOCADO } \\
S \\
(N) \\
\end{array}$ & $\begin{array}{l}\text { COMPARECIMENTOS } \\
(\mathrm{N})\end{array}$ & $\begin{array}{l}\text { BLOQUEIO' } \\
\text { OUTRAS } \\
\text { AÇOOES } \\
\text { (N) } \\
\end{array}$ & $\begin{array}{l}\text { COMUNICANTES } \\
\text { CONVOCADOS } \\
\text { (N) }\end{array}$ & $\begin{array}{l}\text { COMPARECIMENTOS } \\
(\mathrm{N})\end{array}$ & $\begin{array}{c}\text { ALTAS } \\
\text { NA } \\
\text { VISITA } \\
\text { N } \\
\end{array}$ \\
\hline Tracoma & 19 & 18 & 15 & 5 & $\cdot$ & 10 & 1 & 3 \\
\hline DPC (1) & 211 & 190 & 170 & 114 & . & - & . & 19 \\
\hline Raiva & 104 & 78 & 16 & 6 & - & . & - & 64 \\
\hline $\mathrm{RN}(2)$ & 14 & 11 & 8 & 5 & - & - & - & 3 \\
\hline Esquis (3) & 5 & 5 & 5 & 5 & - & - & . & - \\
\hline $\mathrm{TBC}(4)$ & 154 & 112 & 86 & 44 & - & 54 & 10 & 10 \\
\hline Hepatite & 3 & 3 & 1 & 0 & 1 & - & - & 1 \\
\hline Rubéola & 1 & 1 & . & - & 1 & - & . & 1 \\
\hline Lues. (5) & 2 & 2 & 2 & 2 & - & - & - & . \\
\hline Meningite & 1 & 1 & - & . & 1 & - & - & - \\
\hline $\mathrm{MH}(6)$ & 4 & 3 & 2 & 2 & . & . & - & 1 \\
\hline Dengue & 2 & 2 & . & . & 2 & . & - & - \\
\hline P10 (7) & 6 & 5 & 3 & 2 & . & - & - & 2 \\
\hline Pediatria Geral & 25 & 19 & 10 & 6 & . & - & . & 9 \\
\hline RN/Puerp & 36 & 30 & 20 & 15 & - & . & . & 10 \\
\hline Varicela & 1 & 1 & - & - & 1 & - & - & - \\
\hline Sarampo & 6 & 5 & - & - & 5 & - & . & - \\
\hline Adulto & 1 & 1 & 1 & 1 & - & - & - & - \\
\hline Leptospi-rose & 1 & 1 & - & - & - & - & - & 1 \\
\hline Vacin (8) & 1 & 1 & - & - & . & - & . & 1 \\
\hline Total & 597 & 489 & 339 & 207 & 11 & 64 & 11 & 125 \\
\hline
\end{tabular}

1. DPC - desnutrição; 2. RN - recém-nascido; 3. esquist - esquistossomose; 4. TBC-tuberculose (doença/suspeita); 5. Lues - sífilis congênita; 6. MH - hanseníase; 7. P10 - criança abaixo do percentil 10; 8 . vacin - reação vacinal 


\section{AVALIAÇÕES}

A avaliação realizada pelos residentes e docentes foi inserida na avaliação geral do estágio de Pediatria Social, o que ocorreu em dois momentos: no meio e no final do estágio.

Nas avaliações realizadas pelos residentes, o estágio de Vigilância à Saúde foi considerado bastante enriquecedor, trazendo uma contribuição significativa para sua formação como pediatra geral. Os objetivos propostos foram atingidos por intermédio do conjunto das atividades realizadas. Dentre os aspectos positivos, eles enfatizaram a riqueza das reflexões sobre os limites e possibilidades de sua prática, propiciadas pelo contato in loco com as condições de vida das pessoas e a construção de uma avaliação crítica da relação entre o serviço de saúde e a população, a partir da visão dos usuários, permitindo-lhes formular sugestões para melhorar a acessibilidade ao mesmo. Entre os problemas citados, mereceu destaque a situação de medo que alguns vivenciaram ao entrar nas favelas e as dificuldades para localizar muitas residências, devido à forma desorganizada como essas áreas habitacionais são formadas e também a falhas de anotação nos prontuários da UBS, muitas vezes incompletos ou desatualizados.

A avaliação realizada pelos docentes do setor de Pediatria Social foi baseada nos depoimentos e avaliações dos próprios residentes e no acompanhamento contínuo das atividades realizadas. Até o presente momento, a experiência da proposta de Vigilância à Saúde foi considerada altamente positiva com relação aos objetivos pretendidos. A tomada de decisões para superar dificuldades e realizar os ajustes necessários ocorreu após discussões com os profissionais do setor envolvidos diretamente na proposta.

A avaliação feita pela Unidade Básica de Saúde onde a proposta tem-se desenvolvido foi boa, uma vez que forneceu subsídios para os profissionais refletirem sobre sua prática e repensarem estratégias de intervenção no nível daquela comunidade. A apresentação periódica dos relatórios de atividades dos residentes na reuniões da equipe da Unidade contribuiu para reavaliar questões organizacionais e de planejamento.

\section{COMENTÁRIOS FINAIS}

A viabilidade deste tipo de proposta, cuja dimensão extrapola o âmbito estritamente acadêmico, envolvendo serviços de saúde de uma rede hierarquizada, com suas deman- das específicas, sua rotina de trabalho e compostos por equipes multidisciplinares, tem como pré-requisito básico a existência de um compromisso ideológico entre os profissionais inseridos nas diferentes instituições, de ensino e de prestação de serviços, no que diz respeito à melhoria da qualidade dos serviços públicos em geral e de saúde especificamente, no sentido de viabilizar um Sistema Único de Saúde universal, igualitário e de boa qualidade. Esta identificação com um objetivo comum, apesar das diferentes inserções institucionais, possibilita um bom relacionamento entre os profissionais, condição fundamental na concretização de propostas deste tipo, nas quais as dificuldades são inúmeras e a dedicação e o engajamento realmente fazem uma grande diferença quando se busca superar um modelo pedagógico ainda hoje hegemônico nas escolas médicas.

\section{SUMMARY}

One of the most important focus on the reform of medical education in the last decades has been the integration between teaching and health services, bringing contributions to the construction of a new health care model and, at the same time, offering the university a field of research and teaching. The authors report a teaching experience in Health Surveillance, through the insertion of Pediatrics residents in a health care net, where they perform health care to children and adolescents, supervised by a teacher. This training was reformed several times since its implementation, following the process of changings in the health care system and aiming to improve the teaching-learning proposals. The authors report the proposal's objective in the training, describe the pedagogic strategies used, present the outcomes of the activities performed and anlyze its impact on both teaching and health service, with criticisms and suggestions for improvement.

\section{KEYNWORDS}

- Health Surveillance;

- Clinical clerkship-trends;

- Medical staff hospital;

- Pediatric assistants - education. 


\section{REFERÊNCIAS BIBLIOGRÁFICAS}

1. CALDAS, J. R.; BERTONCELLO, N. M. F.; SOCORRO, M.; LINS, A. M.; CYRINO, A. P. P.; TREZZA, E. M. C.; CYRINO, E. G.; CORREA, F. K.; MACHADO, J. L. C. O ideário UNI e a formação e capacitação de recursos humanos: processos e resultados. Divulgação em Saúde para Debate, $\mathrm{n}$. 77-89, CEBES, Jul. 1996.

2. MENDES, E. V. A Vigilância à Saúde no Distrito Sanitário. Série Desenvolvimento de Serviços de Saúde, n. 10: 7-19, OPS, Brasília, 1993.

3.NETO, E. R. SUS, o ensino médico e os hospitais universitários. Saúde em Debate, n. 7-8, CEBES, Dez.1995-Mar.1996.

4. LIPKIN, M.; QUILL, T. E.; NAPODANO, R. J. The medical interview: a core curriculum for residencies in internal medicine. Annals of Internal Medicine, n. 100: 277, 1984.

5. TRESOLINI, C. P. \& SHUGARS, D. A. An integrated health care model in medical education: interviews with faculty and administrators. Acad Med, n. 69 (3): 231-6, 1994.

6. GEZAIRY, H. The main challenges. Medical Education , n.29 (Supplement): 24-7, 1995.

7. DONSKY, J.; VILLELA, T.; RODRIGUEZ, M.; GRUMBACH, $\mathrm{K}$. Teaching community-oriented primary care through longitudinal group projects. Fam Med , n. 30(6): 424-30, 1998.

8. MARCONDES, E.; FUJIMURA, I.; MASCARETTI, L. A.; VANZOLINI, M. E. Opinion of the people in charge of medical graduation disciplines at the Sao Paulo University Medical School on the curriculum evaluation program. Rev Hosp Clin Fac Med Sao Paulo, n. 50(2): 129-32, 1995.

9. STONE, S. L. \& QUALTERS, D. M. Course-based assessment: implementing outcome assessment in medical education. Acad Med, n. 73(4): 397-401, 1998.

10. WILKES, M. S.; USATINE, R.; SLAVIN, S.; HOFFMAN, J. R. Doctoring: Universtiy of California, Los Angeles. Acad Med, n. 73(1): 32-40, 1998.

11. BLOOM, S. W. Structure and Ideology in Medical Education: An Analysis of Resistance to Change. Journal of Health and Social Behavior, n. 29:294-306,1998.

12. PAIM, J. S. \& TEIXEIRA, M. G. L. C. Reorganização do Sistema de Vigilância Epidemiológica na Perspectiva do Sistema Único de Saúde (SUS). Informe Epidemiológico do SUS, 27-55, CENEPI, Out.1992 .
13. WHO The Sociology of Professional Training and Health Manpower: A Summary Report. Working Party on Social Science in Medical Education. Geneva,1972.

14. NETO, E. R. Reorientando o setor saúde no Brasil: desafios para a prática e a educação médicas. Divulgação em Saúde para Debate, n.14: 66-74, CEBES, Ago1996.

15. ARAUJO, E. C.; TEIXEIRA, C. F.; RANGEL, M. L. Relação ensino-serviço-comunidade: a contribuição da rede IDA na reorientação do ensino e das práticas de saúde no SUS. Saúde em Debate, n. 49-50: 88-94, CEBES, Dez 1995-Mar 1996.

16. FEUERWERKER, L. C. M. \& MARSIGLIA, R. Estratégias para mudanças na formação de rhs com base nas experiências IDA / UNI. Divulgação em Saúde para Debate, n. 12: 24-28, Jul 1996.

17. BELACIANO, M. I. O SUS deve aceitar este desafio: elaborar proposições para a formação e capacitação de recursos humanos em saúde. Divulgação em Saúde para Debate , n.12: 29-33, CEBES, Jul 1996.

18. CINAEM. Transformando a educação médica brasileira: Projeto CINAEM - III Fase. mimeo 81 p, 1998.

19. CYRINO, E. G. O diagnóstico de saúde da criança no ensino médico: relato de uma experiência. Divulgação em Saúde para Debate, n.11: 20-5, CEBES, Set 1995.

20. TREZZA, E. M. C. \& PREARO, A. Y. Saúde da criança: curso de graduação médica em centros de saúde, creches e escola. Divulgação em Saúde para Debate , n.11: 26-9, CEBES, Set 1995.

21. YAMAMOTO, R. M.; PRIMO, E.; CARDOSO, I.; CONCEIÇÃO, E. M.; ANZAI, I. M. N.; SANTOS, M. G.; OLIVEIRA, N. L.; LEONE, C. Um modelo de ensino para médicos residentes na área de Pediatria Comunitária: a visita domiciliar contribuindo para uma formação profissional mais abrangente. Pediatria (São Paulo), n.20 (3): 172-8, São Paulo, 1998.

22. TEIXEIRA, C. F.; PAIM, J. S.; VILASBÔAS, A. L. SUS, modelos assistenciais e vigilância da saúde. Informe epidemiológico do SUS , n.VII (2): 7-28, Brasília, 1998.

23. DOE-DIÁRIO OFICIAL DO ESTADO DE SÃO PAULO. Lei complementar $n^{\circ} 791 / 95$. 
Endereço para correspondência

Selma Cristina Franco

Rua João Brásio,175

13092-510 - Campinas - São Paulo

Tel.: (019) 251-2059

E-mails:

Selma Cristina Franco

sfranco@obelix.unicamp.br

Emília de Faria Carniel emilia_carniel@zipmail.com.br Maria Ângela R. G. M. Antônio slantoni@correionet.com.br

Maria de Lurdes Zanolli

morcillo@obelix.unicamp.br 